\title{
Reflexión sobre un problema profesional en el contexto de formación de profesores
}

\section{A reflection on a professional problem in the context of teacher education}

\section{Juan Luis Piñeiro ${ }^{1}$ \\ Pablo Flores ${ }^{2}$}

\begin{abstract}
Resumen. Este trabajo tiene como objetivo describir un ciclo de reflexión realizado en un curso sobre desarrollo profesional y el conocimiento del profesor de matemáticas, correspondiente a la Maestría en Educación Matemática de la Universidad de Granada. La reflexión se inicia desde un problema profesional y se realiza bajo los enfoques teóricos de Smith (1991), desde la perspectiva del estudiante/formador. Describimos el proceso realizado a lo largo del curso, haciendo evidente el proceso de cambio ocurrido. Finalmente discutimos algunas de las posibles contribuciones de este ciclo en la promoción del desarrollo profesional de los formadores de profesores.
\end{abstract}

Palabras clave: reflexión para la enseñanza, desarrollo profesional, profesor de matemáticas, formación de profesores.

Abstract. This paper aims to describe a cycle of reflection carried out in a course on professional development and knowledge of the mathematics teacher, belonging to the Master in Mathematical Education of the University of Granada. Reflection starts from a professional problem and is carried out under the

Fecha de recepción: 22 de marzo de 2017. Fecha de aceptación: 12 de septiembre de 2017

1 Doctorando, Universidad de Granada, España, juanluis.pineiro@gmail.com

2 Departamento de Didáctica de la Matemática, Universidad de Granada, España, pflores@ugr.es 
theoretical approaches of Smith (1991), from the perspective of the student / trainer. We describe the process carried out along the course. Finally we discuss some of the possible contributions of this cycle can promote on professional development of teacher trainers.

Keywords: teaching reflection, professional development, math teacher, teacher training.

\section{INTRODUCCIÓN}

La actividad reflexiva del profesor de matemáticas sobre sus prácticas debería constituir parte de su proceso de formación (Schön, 1992). Dicha actividad brinda la posibilidad de tomar distancia del problema profesional, dando espacio para contemplar la actuación en la sala de clases (Brubacher, Case y Reagan, 2000; Flores, 1998, 2007; Jaworski, 1993). Bajo estos supuestos, se han desarroIlado modelos teóricos que promueven la reflexión sobre la práctica (e. g. Korthagen, 2010), en ellos se enfatizan los beneficios de promover los procesos reflexivos y se hace notar la complejidad que acarrea. Junto a esta dificultad, se debe tener en cuenta que convertirse en un profesor reflexivo es un proceso a largo plazo.

Por todo esto, es de esperar que las dificultades manifestadas por los profesores, en formación o en activo, al momento de reflexionar se transfieran a los formadores de profesores. Block y colaboradores (2013) muestran, en un contexto de profesores asesores, que la reflexión de la práctica puede promover cambios en las decisiones que toman los formadores para acompañar a los futuros docentes o los procesos de mejoramiento. Además, estos autores hacen evidente que los profesores y los formadores tienen miradas distintas sobre qué reflexionar. Reafirmando esta idea, Chapman (2009) señala que la reflexión en el proceso de aprender a enseñar juega un rol fundamental.

En este contexto, describir procesos de reflexión en instancias de preparación de formadores daría información útil sobre las condiciones en que se producen la reflexión del formador. Una de las instancias para formar a los profesores universitarios son los posgrados. Por tanto, nos planteamos como objetivo de este trabajo, mostrar un ciclo reflexivo realizado en el contexto de una asignatura del Máster en Didáctica de la Matemática de la Universidad de Granada, en España. Dicho ciclo fue hecho por un formador de profesores novato, desde un 
problema de su propia práctica. Para esto, primero se enmarcan teóricamente las discusiones, posteriormente se describe el ciclo utilizado, terminando con reflexiones sobre el proceso.

\section{LA REFLEXIÓN Y EL DESARROLLO PROFESIONAL DOCENTE}

La reflexión del profesor es un tema de interés dentro de la Didáctica de la Matemática, que se materializa en la gran cantidad de trabajos presentados en los diferentes encuentros de investigadores del área y en publicaciones como el "International Handbook of Mathematics Teacher Education" del año 2008 (Wood, 2008).

La reflexión se relaciona estrechamente con el desarrollo profesional de un profesor, considerándose un aspecto que lo favorece. En este sentido, se entiende a la reflexión como una imbricada vinculación entre técnicas y experiencias, que hacen emerger posibles herramientas que permitirán una toma de decisiones justificada (Brubacher et al., 2000). Esta relación se hace visible de numerosas formas, sin embargo la acción más concreta es la de mirar atrás, metacognitivamente, para revisar la práctica.

La conceptualización del profesor como un profesional reflexivo se inspira en las ideas de Dewey (1989). Este autor define la reflexión cómo la mejor forma de pensar, que surge desde la necesidad de resolver un problema y la describe cómo una secuencia dinámica en la que las ideas se relacionan entre sí. Estas ideas, tienen por objeto establecer una conclusión, es decir, tienen una meta. Flores (2007), además, hace notar que llevar a cabo un proceso reflexivo para resolver problemas de la práctica, contribuye al desarrollo profesional del profesor de matemáticas.

Schön (1992), al esclarecer las diferencias entre racionalidad teórica y racionalidad práctica, promueve que se comience a usar la reflexión para describir una cualidad deseada en los profesores. Flores (2007) aclara las ideas de Shön de una forma muy concisa, al exponer que "el conocimiento del profesional reflexivo no precede a la acción, sino que está implicado con el conocimiento práctico, que deriva y está en la acción misma" (p.142). Esto implicaría reflexión no solo para, sino en y sobre la acción realizada en el aula.

Killion y Todnem (1991) ahondan en estos tipos de reflexión, partiendo desde las ideas de Schön, y mediante ejemplos en un taller realizado con profesores. En sus planteamientos, la reflexión en la acción es la realizada durante la 
práctica, y se realiza sobre los fenómenos y en las propias formas espontáneas de pensar y actuar en medio de la acción. Mientras que la reflexión sobre la acción afecta acciones y pensamientos personales, y se lleva a cabo después que se completó la práctica. Finalmente, profundizan en un tipo que es el resultado de los dos tipos anteriores: reflexión para la acción. Estos autores la describen como "el resultado deseado de los dos primeros tipos expuestos. Reflexionamos, no tanto para revisar el pasado o para tomar conciencia del proceso metacognitivo que estamos experimentando (dos nobles razones, indudablemente), sino para guiar la acción futura (el propósito más práctico)" (p. 15). Bajo esta misma premisa, se puede inferir que los profesores principiantes realizan sobre todo la reflexión para y sobre la práctica; mientras que los profesores expertos pueden llevar a cabo la reflexión en la práctica (Brubacher et al., 2000).

Esta idea procesual de reflexión en el desarrollo profesional del profesor se completa con las ideas de van Manen (1977), quien se ocupa del nivel de profundidad que puede abarcar la reflexión. Este autor postula un modelo que consta de tres niveles jerarquizados. El primer nivel se describe como la aplicación eficaz de técnicas y criterios educativos. El segundo nivel, más avanzado, se corresponde con un tipo que implica las consecuencias de la aplicación de determinadas estrategias, currículos o acciones propias del quehacer educativo. Finalmente, el nivel más profundo tiene que ver con la posibilidad de pensar críticamente sobre los elementos educativos planteados por el sistema.

En este enfoque se observa como existen elementos sobre los que se reflexiona. Sparks-Langer y Colton (1991), sistematizando las investigaciones sobre pensamiento reflexivo en profesores, describen tres elementos que configuran significativamente esta noción. El primero, se refiere a los elementos cognitivos o de conocimiento profesional del profesor. Un segundo elemento, considerado crítico, agrupa los elementos morales y éticos referidos a los procesos educativos en los que se desenvuelve el docente. Finalmente, un tercer elemento, de carácter concreto, son las narraciones; entendidas como la capacidad de exponer y verbalizar sus experiencias.

Bajo estas miradas revisadas y otras más, Ramos-Rodríguez, Rojas y Flores (2013), realizan una caracterización del profesor de matemáticas reflexivo, concretando en Ramos-Rodríguez (2014) lo que se entiende por reflexión en este trabajo. Traduciéndose en la disposición:

A percibir situaciones del entorno que requieren una actuación personal de su parte. 
A distanciarse de ellas para poder analizar sus elementos.

A explicitar y eliminar elementos que condicionan la forma de percibir esas situaciones, incluidos los derivados de sus creencias o esquemas implícitos.

A recurrir a otras fuentes para buscar otras formas de interpretar las situaciones y de responder a las mismas.

A obligarse a traspasar los límites de su zona de bienestar: la zona con la que está familiarizado y en la que se siente a salvo (Korthagen, 2010).

A dar significado a su acción, a tomar conciencia de la complejidad de la práctica y del aprendizaje de sus alumnos, otorgándole sentido a su práctica profesional (Climent, 2005).

A adaptar su actuación práctica a las condiciones del contexto, como por ejemplo, tomar en consideración la particularidad de su situación, de los alumnos a los que se dirige, de su individualidad, etc. (Cooney, 2001).

A abrirse hacia las matemáticas y a fundamentar sus concepciones sobre ella.

A tomar conciencia de la complejidad de la matemática para su enseñanza. (Ramos-Rodríguez, 2014, pp.21-23)

Las nociones presentadas son exigentes y propician procesos de desarrollo profesional continuo y sistemático desde la formación inicial. Por tanto, esta instancia debería entenderse y configurarse en torno a estas nociones.

Para llevar a cabo procesos de reflexión se han propuesto numerosos modelos. Uno de ellos es el elaborado por Smith (1991) y que consta de 4 fases o etapas, que se detallan en la figura 1.

Para este autor, el proceso reflexivo surge desde la necesidad de generar cambios positivos en las escuelas, pero desde sus bases, es decir, desde la percepción de un problema profesional realizado por el profesor. Por tanto, en cada una de las fases, la actuación del docente es fundamental. Este modelo se origina en procesos de formación continua, por ello se presenta como una buena opción al momento de formar investigadores en Didáctica de la Matemática que dedican una asignatura al desarrollo y al conocimiento profesional del profesor. Existen antecedentes sobre cómo este ciclo reflexivo muestra sus frutos en profesores de matemáticas (Flores y Fernández, 2001; Nancupil, Carneiro y Flores, 2013; Peñas, 2003; Peñas y Flores, 2005). En este trabajo se describe un ciclo de reflexión desde la perspectiva del formador. 


\begin{tabular}{|c|c|}
\hline 1. Descripción & 3. Confrontación \\
\hline $\begin{array}{l}\text { “¿Cuáles son mis practicas?” } \\
\text { Señalar ejemplos de prácticas } \\
\text { que reflejen: } \\
\text { - } \text { Regularidades } \\
\text { - } \text { Contradicciones } \\
\text { - } \text { Hechos relevantes } \\
\text { - } \text { Hechos irrelevantes } \\
\text { Incluyendo elementos de: } \\
\\
\quad \text { ¿quién? } \\
\quad \text { ¿qué? } \\
\text { ¿cuándo? }\end{array}$ & $\begin{array}{c}\text { “Cuáles son las causas?” } \\
\text { - ¿Supuestos, valores, } \\
\text { creencias? } \\
\text { - ¿De dónde proceden? } \\
\text { - ¿Qué prácticas sociales } \\
\text { expresan? } \\
\text { - ¿Qué es lo que mantiene } \\
\text { mis teorías? } \\
\text { - ¿Qué es lo que encierran } \\
\quad \text { mis teorías? } \\
\text { - ¿Qué relación existe entre } \\
\text { lo personal y lo social? } \\
\text { - ¿Qué intereses están } \\
\text { siendo servidos? }\end{array}$ \\
\hline 2. Inspiración & 4. Reconstrucción \\
\hline $\begin{array}{l}\text { “Qué teorías expresan mis } \\
\text { prácticas?” } \\
\text { Analizar las descripciones para } \\
\text { intentar determinar las } \\
\text { relaciones existentes entre los } \\
\text { distintos elementos y en } \\
\text { función de esto, hacer una serie } \\
\text { de afirmaciones del tipo: } \\
\text { "parece como si...” }\end{array}$ & $\begin{array}{l}\text { “Cómo podría cambiar?” } \\
\text { - ¿Qué podría hacer } \\
\text { diferente? } \\
\text { - ¿Qué es lo que considero } \\
\text { importante desde un } \\
\text { punto de vista } \\
\text { pedagógico? } \\
\text { - ¿Qué es lo que tendría } \\
\text { que hacer para introducir } \\
\text { estos cambios? }\end{array}$ \\
\hline
\end{tabular}

Figura 1. Ciclo de reflexión (Smith, 1991, p.280)

Durante dicho curso se invitó a los estudiantes a describir un problema profesional relacionado con la práctica. A partir de la experiencia previa, se decidió plantear un problema relacionado con la actuación como formador de profesores, en la Universidad Metropolitana de Ciencias de la Educación, en Chile. A continuación se describe el proceso llevado a cabo, indicando las etapas que se recorrieron, de acuerdo con el modelo propuesto por Smith (1991). 


\section{CICLO DE REFLEXIÓN}

El ciclo de reflexión de Smith (1991), está compuesto de cuatro fases o etapas: a) descripción, b) inspiración, c) confrontación y d) reformulación. A continuación, se muestra este proceso, enfatizando en los aspectos relativos a la reflexión realizada por el formador.

\section{DESCRIPCIÓN}

Un primer requisito para la actitud reflexiva es percibir la práctica docente como problemática. Por tanto un primer paso de este ciclo es percibir las problemáticas de la práctica docente, para lo cuál es de utilidad ser capaz de "describir nuestras prácticas y (a) utilizar dichas descripciones como base para posteriores debates y desarrollos" (Smith, 1991, p. 282). En esta primera etapa se realizó la definición de una situación que emergía como un problema profesional. Para describirlo, en una primera fase se redactó en forma de narración, tratando de aclarar lo más posible. En este caso, se explicó, que el contexto fue en el transcurso de la asignatura Didáctica de Sectores, carrera de pedagogía, en una universidad chilena. Los estudiantes se mostraron muy receptivos al modelo de enseñanza aproximativo planteado (Charnay, 1994); mostrando reflexiones coherentes con lo expuesto por el autor y rechazando una enseñanza puramente procedimental de las matemáticas. Sin embargo, cuando se observan prácticas en las aulas, se aprecia que los estudiantes manifiestan un modelo normativo en su actuar. En términos de Charnay (1994), se corresponde con tomar un rol docente de expositor, característica que predomina en los futuros profesores, en el que incluso no permiten responder a sus estudiantes, haciéndolo ellos mismos sin dar tiempo de pensar una respuesta.

Para abordar y comprender el problema, se focalizó a través de las tres interrogantes que el autor destaca como determinantes en relación al problema práctico destacado: ¿quién?, ¿qué? y ¿quiénes? son los afectados. Aclarar estos aspectos, genera los elementos que se muestran en la tabla 1. 
Tabla 1. Definición del problema profesional

\begin{tabular}{ll}
\hline Problema & \\
\hline & Nivel: Universitario \\
& Carrera: Pedagogía \\
Contexto & $\begin{array}{l}\text { Tercer año de carrera. VI semestre. } \\
\text { Uso de la resolución de problemas en clases de matemáticas. } \\
\text { Problemas aritméticos de enunciado verbal. }\end{array}$ \\
\cline { 2 - 3 } Déficit & Los estudiantes tienen un discurso teórico muy rico sobre la importancia y el \\
& rol de la resolución de problemas en las clases de matemáticas, pero al \\
& observar sus clases prácticas, se aprecia que aplican un modelo normativo \\
& de la enseñanza, sólo usan los problemas como instrumento de evaluación. \\
\cline { 2 - 3 } Problema & ¿Cómo enseñar a los futuros profesores para que comprendan el rol de la \\
& resolución de problemas aritméticos aditivos en clases de matemáticas? \\
\hline
\end{tabular}

\section{INSPIRACIÓN O INFORMACIÓN}

La sola descripción de una situación que se considera problemática no es suficiente. Una vez realizado el ejercicio de definir y describir claramente el problema en la enseñanza inicial de profesores, se debe avanzar a un segundo estadio. La fase siguiente corresponde a la de información o inspiración. En palabras de Smith (1991), busca "¿cuál es el sentido de mi enseñanza?" (p. 282). El objetivo de esta fase es aclarar las creencias propias sobre "leyes universales que rigen la enseñanza" (p. 282). Esto se traduce en encontrar cuáles son los marcos teóricos que mueven o inspiran la acción que hizo actuar de determinada forma, dando origen al problema profesional.

Existen diversas estrategias para favorecer este distanciamiento. En este contexto se realizó mediante la colaboración de los compañeros del curso, quienes enunciaron creencias que apreciaban en el planteamiento del problema. A partir del reactivo yo creo que tú crees... surge uno de los elementos más significativos. Las aportaciones de los compañeros llevaron a examinar como creencia propia que la resolución de problema es una metodología transversal a cualquier contenido matemático, sin ver la complejidad de las diferentes maneras de trabajarlo. El estudiante/formador pensaba que cualquier profesor lo trae internalizado, sin tomar en cuenta las teorías detrás de la resolución de problemas. Estas palabras provocaron la necesidad de revisar 
cómo se conceptualiza la resolución de problemas, en el papel de formador de profesores. La colaboración de los compañeros permitió reconocer que, efectivamente el discurso hasta ese momento se dirigía a entender la resolución como una metodología a aplicar y no a considerarla en la complejidad de su tratamiento en el aula.

Por otro lado, y en una mirada general sobre cada creencia expuesta por los pares, se observa la apropiación de un modelo aproximativo del conocimiento (Charnay, 1994) desde dónde surge la problemática. Este se propone partir de modelos, de concepciones existentes en el alumno y ponerlas a prueba para mejorarlas, modificarlas o construirlas; el profesor propone y organiza una serie de situaciones con distintos obstáculos, organiza las diferentes fases de investigación, formulación, validación e institucionalización; organiza la comunicación de la clase, propone en el momento adecuado los elementos convencionales del saber (notaciones, simbologías); el alumno ensaya, busca, propone soluciones, las confronta con la de sus compañeros, las defiende o las discute. El saber es considerado con su lógica propia. Esta serie de descriptores permiten ver la complejidad de enseñar la resolución de problemas, y muestran algunas creencias, que hacen notar la ingenuidad con que se había entendido el acto de aprender matemáticas por medio de la resolución de problemas. Van de Walle, Karp y Bay-Williams (2013) y más recientemente, Castro y Ruíz-Hidalgo (2015) hacen un completo recorrido por los diferentes conceptos que un profesor debiera conocer. En ambos casos, queda en evidencia el gran número de conocimientos que se deben poner en juego en un acto pedagógico que utilice la resolución de problemas y la intrincada relación que debe darse entre ellos.

\section{CONFRONTACIÓN}

Una vez indagado en las teorías subyacentes a la práctica, se está en disposición de reflexionar con otros o a la luz de aportes teóricos. Este ciclo de reflexión se realizó, principalmente, por el último camino mencionado. Smith (1991) lo describe a través de la pregunta "¿Cómo llegué a ser de este modo?" (p. 285). Con su respuesta, se espera confrontar la posición de quien realiza el ciclo de reflexión con sus creencias y convicciones, mostrando las ideas que nos llevaron a actuar de esa forma. En el curso, esta fase comenzó con los aportes entre pares para afrontar los problemas planteados, sugiriendo textos que trataran aspectos de dicho problema. De estos documentos, así como de una búsqueda más 
sistemática sobre la importancia de la resolución de problemas en la enseñanza de las matemáticas y los elementos que la configuran, surgen las apreciaciones que se describen a continuación.

La creencia de que no hay nada más básico en una disciplina que su modo de pensar (Charnay, 1994), lleva a plantearse como un problema, el que los futuros profesores sean capaces de enseñar por medio de la resolución de problemas y todo lo que eso significa. Sin embargo, apreciamos que es ingenuo simplificarlo a un nivel de metodología o estrategia. Resolver problemas muchas veces se asocia con enseñar a pensar (Mayer, 1986), por tanto, al enseñar a resolver problemas, se está enseñando a pensar; pero este pensamiento tiene unas características especiales: es crítico, creativo y metacognitivo (Beas, Santa Cruz, Thomsen y Utreras, 2001). Bajo esta premisa, y por el contexto de formación, es necesario identificar qué elementos de conocimiento profesional requieren los profesores para realizar una enseñanza efectiva de la resolución de problemas en el aula.

En este contexto, aún no existe una sistematización sobre qué debe saber un profesor para enseñar la resolución de problemas. Chapman (2015) realiza un primer intento partiendo del modelo Mathematical Knowledge for Teaching (MKT) (Ball, Thames y Phelps, 2008). En esta sistematización, se recurrió a las principales investigaciones en torno a la resolución de problemas. De acuerdo con esta autora, la enseñanza de la resolución de problemas es un sistema que se relaciona estrechamente (ver figura 2). El marco de Chapman (2015) se compone de la competencia del profesor para resolver problemas, conocimientos del contenido (sobre problemas, resolución e invención de estos) y didácticos del contenido (sobre los estudiantes como resolutores y prácticas de enseñanza). Además, en una dimensión aparte, se encuentran los factores afectivos y creencias que tienen incidencia en la enseñanza de esta competencia.

Sin embargo, esta primera aproximación no pretende ser definitiva, pues como la misma autora plantea, existe un conocimiento o experiencia propia, del futuro docente, que no se clasifica como conocimiento. En Piñeiro, Castro y Castro-Rodríguez (2016) se realiza una descripción sobre este mismo constructo, pero desde una perspectiva curricular. En general, es bastante coincidente, destacando el carácter sistémico y complejo, con un nivel práctico y teórico; y dimensiones personales y profesionales.

Con esto queda claro, que la dificultad que se expresa en la sala de clases para enseñar la resolución de problemas es un tema que está lejos de agotarse y esclarecerse. No obstante, este ciclo de reflexión permite apreciar algunas 
dimensiones que, junto con mostrar la complejidad que implica enseñar a resolver problemas, aportan nuevas perspectivas clarificadoras.

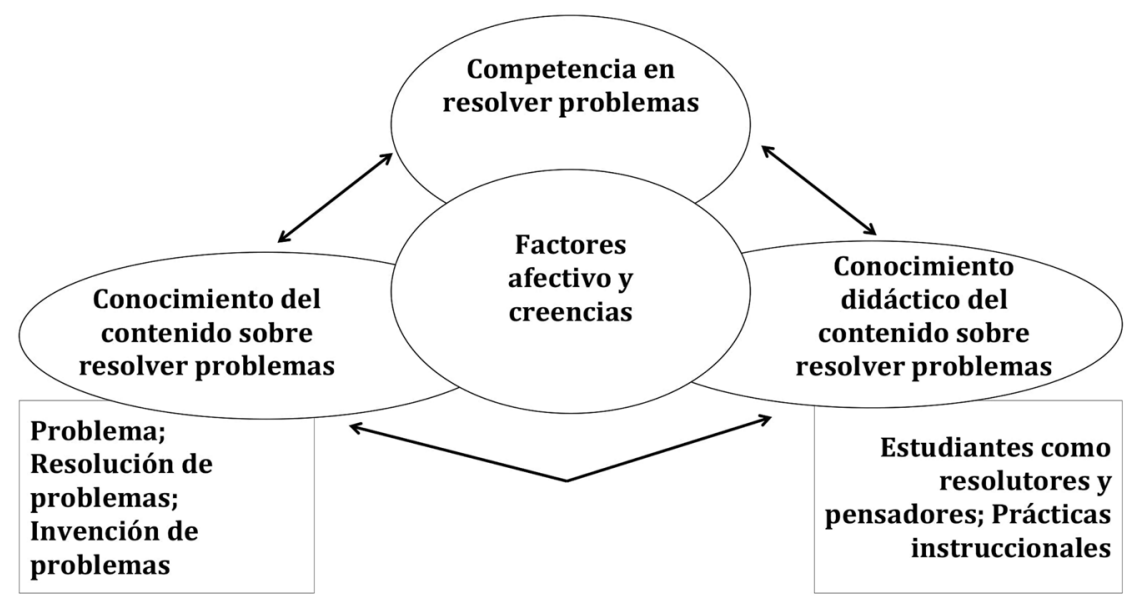

Figura 2. Relaciones del Modelo de conocimiento para la enseñanza de la resolución de problemas (Chapman, 2015, p. 32)

\section{REFORMULACIÓN}

Una última fase de este ciclo corresponde a la reformulación. Smith lo describe con la pregunta "¿Cómo podría hacer las cosas de otro modo?" (p. 291). Es aquí dónde se podrá ver el resultado final de tomar conciencia de nuestras ideas y nuestras prácticas.

La fase de confrontación se realiza a partir de las lecturas hechas durante la fase anterior, en este caso dio luces sobre la complejidad de la enseñanza de la resolución de problemas. Se aprecia que el problema planteado en un inicio es importante en el área, y su solución no es fácil y directa, por ello la reformulación se realizó en este contexto. La caracterización de Chapman (2015) y la creencia que simplificaba la resolución de problemas a una metodología, son opuestas. Es así como desde la preocupación de la enseñanza de la resolución de problemas, se ha avanzado a una preocupación por enseñar la mayor cantidad de aspectos que podrían influir en la enseñanza exitosa de la resolución de problemas; además se considera también el papel de las concepciones y creencias, y la propia competencia para resolver problemas. Por tanto, en el mismo contexto que se definió 
el problema, se aborda el nuevo: cómo lograr que los futuros profesores aprecien los distintos roles que puede jugar la resolución de problemas en la enseñanza de la matemática, cómo hacer que aprecien la diferencia entre su consideración como una metodología de enseñanza, y un procedimiento que mide el rendimiento matemático, entre otros.

\section{REFLEXIONES FINALES}

La reflexión es un elemento primordial para el desarrollo profesional (Flores, 2007; Jaworski, 1993). Sin embargo, por diversos factores, no es una práctica cotidiana en las salas de profesores, consejos escolares o reuniones docentes. En el ciclo de reflexión descrito en este trabajo, se observan las numerosas ventajas de realizar una práctica reflexiva, especialmente cuando la pone en juego el propio formador de profesores. La potencialidad de desarrollar profesionalmente a futuros formadores es exponencial por el impacto que tendrá en cientos de profesores en pocos años.

Este ciclo ha permitido identificar algunos de estos beneficios, entre los cuales destacamos: a) la potenciación de un trabajo en equipo, coincidente con los expuesto por Alsina (2007), quién al presentar un modelo de formación eficaz, plantea que la reflexión y las comunidades de aprendizaje e indagación son fundamentales, b) ayuda a disminuir la confrontación teoría-práctica, una cuestión que Godino (1991) expone como una dificultad entre la academia y los profesores, c) ampliar la visión sobre el tema problemático, visualizándolo de manera global y sistémica, d) confrontar las creencias con la práctica, y claro, e) mejorar nuestras propias prácticas, y por consiguiente, la calidad de la educación que reciben nuestros estudiantes.

Específicamente en el ámbito de formadores de profesores, el ciclo de reflexión presentado expone un tema transversal de la enseñanza de la matemática, como es la resolución de problemas, por lo que el producto de la investigación de Smith (1991), no solo tiene frutos en la escuela, sino también en la academia.

Junto con esto, es interesante que el formador reflexione sobre qué deberían saber sus estudiantes (futuros profesores) y no sólo sobre aspectos de las tareas proporcionadas o metodologías utilizadas, como muestran experiencias realizadas con docentes de aula (e. g. Ñancupil et al., 2013) 
Por todo esto, es recomendable que actividades de reflexión, sean parte, tanto de la formación inicial y continua, como del desarrollo profesional del formador de profesores. Esto implicaría facilidades para que el docente, en el nivel que sea pueda convertirse en un profesional práctico reflexivo, pues es una meta compleja pero alcanzable, favorecer la transición a un profesional práctico reflexivo, en donde el término reflexivo se entienda en la complejidad entera que implica.

\section{REFERENCIAS}

Alsina, A. (2007). El aprendizaje reflexivo en la formación permanente del profesorado : un análisis desde la didáctica de la matemática. Educación Matemática, 19(1), pp. 99-126.

Ball, D. L., Thames, M. H. y Phelps, G. (2008). Content knowledge for Teaching. What makes itSpecial? Journal of TeacherEducation, 59(5), pp. 389-407. doi.org/10.1177/0022487108324554

Beas, J., Santa Cruz, J., Thomsen, P. y Utreras, S. (2001). Enseñar a pensar para aprender mejor. Santiago, Chile: Ediciones UC.

Block, D., Martínez, P., Mendoza, T. y Ramírez, M. (2013). La observación y el análisis de las prácticas de enseñar matemáticas como recursos para la formación continua de maestros de primaria. Reflexiones sobre una experiencia. Educación Matemática, 25(2), pp. 31-59.

Brubacher, J. W., Case, C. W. y Reagan, T. G. (2000). Cómo ser un docente reflexivo: Ia construcción de una cultura de la indagación en las escuelas. Barcelona, España: Gedisa.

Castro, E. y Ruíz-Hidalgo, J. F. (2015). Matemáticas y resolución de problemas. En P. Flores y L. Rico (Eds.), Enseñanza y aprendizaje de las matemáticas en educación primaria (pp. 89-108). Madrid, España: Pirámide.

Chapman, O. (2009). Educators Reflecting on (researching) their own Practice. In R. Even y D. L. Ball (Eds.), The Professional Education and Development of Teachers of Mathematics. The 15th OCMI Study (pp. 121-126). New York, NY: Springer. doi. org/10.1007/978-0-387-09601-8_14

Chapman, O. (2015). Mathematics Teachers' Knowledge for Teaching Problem Solving. LUMAT, 3(1), pp. 19-36.

Charnay, R. (1994). Aprender (por medio de) la resolución de problemas. En C. Parra e I. Sainz (Eds.), Didáctica de las matemáticas. Aportes y reflexiones (pp. 51-64). Buenos Aires, Argentina: Paidós. 
Dewey, J. (1989). Cómo pensamos: Nueva exposición de la relación entre pensamiento y proceso educativo. Barcelona, España: Paidós.

Flores, P. (1998). Formación inicial de profesores de matemáticas como profesionales reflexivos. UNO, (17), pp. 37-50.

Flores, P. (2007). Profesores de matemáticas reflexivos: formación y cuestiones de investigación. PNA, 1(4), pp. 139-159.

Flores, P. y Fernández, F. (2001). Reflexiones sobre un problema profesional relacionado con la enseñanza del álgebra. En F. J. P. Perales Palacios, A. García, J. Bernal, F. Maeso, J. Muros, L. Rico y J. Roldán (Eds.), Congreso nacional de didácticas especificas (pp. 1787-1800). Granada, España: GEU.

Godino, J. D. (1991). Hacia una teoría de la Didáctica de la Matemática. En Á. Gutiérrez (Ed.), Área de conocimiento Didáctica de la Matemática (pp. 105-149). Madrid, España: Síntesis.

Jaworski, B. (1993). The Professional Development of Teachers. The Potential of Critical Reflection. British Journal of In-Service Education, 19(3), pp. 37-42. doi. org/10.1080/0305763930190307

Killion, J. P. y Todnem, G. R. (1991). A Process for Personal Theory Building. Educational Leadership, 48(6), pp. 14-16.

Korthagen, F. A. J. (2010). La práctica, la teoría y la persona en la formación del profesorado. Revista Interuniversitaria de Formación del Profesorado, (68), pp. 83-101.

Mayer, R. E. (1986). Pensamiento, resolución de problemas y cognición. Barcelona, España: Ediciones Paidós.

Nancupil, J. C., Carneiro, R. y Flores, P. (2013). La reflexión sobre la práctica del profesor de matemática: el caso de la enseñanza de las operaciones con números enteros. Unión: revista iberoamericana de educación matemática, (34), pp. 37-46.

Peñas, M. (2003). Los números enteros y la calculadora. Una experiencia de reflexión sobre la práctica. UNO, (32), pp. 109-118.

Peñas, M. y Flores, P. (2005). Procesos de reflexión en estudiantes para profesor de matemáticas. Enseñanza de las ciencias, 23(1), pp. 5-16.

Piñeiro, J. L., Castro, E. y Castro-Rodríguez, E. (2016). Conocimiento profesional para la enseñanza de la resolución de problemas en primaria: una perspectiva curricular. En J. A. Macías, A. Jiménez, J. L. González, M. T. Sánchez, P. Hernández, C. Fernández, A. Berciano (Eds.), Investigación en educación matemática XX (pp. 427-436). Málaga, España: SEIEM.

Ramos-Rodríguez, E. (2014). Reflexión docente sobre la enseñanza del álgebra en un curso de formación continua. Tesis doctoral. Universidad de Granada, España. 
Ramos-Rodríguez, E., Rojas, N. y Flores, P. (2013). Una aproximación a las nociones de profesor reflexivo y de profesor experto y su repercusión en el docente universitario de matemáticas. En M. T. Ramiro, T. Ramiro y P. Bermúdez (Eds.), X Foro internacional sobre evaluación de la calidad de la investigación y de la educación (FECIES) (pp. 120-125). Granada, España: AEPC.

Schön, D. (1992). La formación de profesionales reflexivos: Hacia un nuevo diseño de la enseñanza y el aprendizaje en las profesiones. Barcelona, España: Paidós.

Smith, J. (1991). Una pedagogía crítica de la práctica en el aula. Revista de Educación, (294), pp. 275-300.

Sparks-Langer, G. M. y Colton, A. B. (1991). Synthesis of Research on Teachers' Reflective Thinking. Educational Leadership, 48(6), pp. 37-44.

Van de Walle, J. A., Karp, K. S. y Bay-Williams, J. M. (2013). Elementary and Middle School Mathematics: Teaching Developmentally (8th ed). Boston, MA: Pearson /Allyn and Bacon.

van Manen, M. (1977). Linking Ways of Knowing with Ways of Being Practical. Curriculum Inquiry, 6(3), pp. 205-228.

Wood, T. (Ed.). (2008). The International Handbook of Mathematics Teacher Education. Rotherdham, Países Bajos: Sense Publishers. 
